\title{
Tribological Property of FG/UHMWPE Composites
}

\author{
Longhua XU11, a, Jiatong SHI' ${ }^{1}$, Yawen $\mathrm{ZHENG}^{1}$, Xingkai ZHANG ${ }^{2}$, \\ Wufanbieke·Baheti1 ${ }^{1}$, Bin LIU¹,b \\ ${ }^{1}$ School of stomatology, Lanzhou University, Lanzhou 730000, China \\ 2 Lanzhou Institute of Chemical Physics, Chinese Academy of Sciences \\ a394226221@qq.com; bliubkq@|zu.edu.cn
}

Keywords: Ultrahigh molecular weight polyethylene; Fluorinated graphene; Tribological properties

\begin{abstract}
Fluorinated graphene/ultra high molecular weight polyethylene (FG/UHMWPE) composites were prepared by ultrasonic dispersion and hot pressing. The mechanical propertys and tribological property of pure UHMWPE and FG/UHMWPE composites were investigated by micro hardness tester and high-speed reciprocating friction tester. The wear volume of the sample was measured by the scanning electron microscope, and Micro-XAM noncontact 3D surface profiler, and the corresponding volume wear rate was also calculated. The results showed adding FG could obviously improve the microhardness of UHMWPE. Adding FG not only reduced the friction coefficient of UHMWPE, but also significantly reduced the volume wear rate of UHMWPE, and the higher the FG content, the smaller the volume wear rate of FG/UHMWPE composites. The addition of FG can improve the tribological property of UHMWPE.
\end{abstract}

\section{Introduction}

Ultrahigh molecular weight polyethylene (UHMWPE) has been widely used in artificial joint for its excellent advantages, such as low friction coefficient and good biocompatibility [1-4]. But UHMWPE has the obvious disadvantages of low Young's modulus and poor wear resistance, which limit its wide application. Therefore, many research works have been done to improve the performance of UHMWPE. One method to improve the wear resistance and mechanical property of the UHMWPE is filling inorganic materials. Carbon nanotubes (CNT), hydroxyapatite, zirconia and alumina served as reinforced filler can improve the wear resistance of UHMWPE to some extent. However, CNT and other reinforcing fillers can reduce the mechanical property, such as tensile strength and impact strength[5-6]. In 2004, by using the method of micro mechanical stripping, Geim Andre prepared graphene. Graphene and its derivatives have attracted much attention because of there unique crystal structure, excellent physical and chemical property, and wide application prospect. Fluorinated graphene (FG) not only has the specific characteristics of graphene, such as the physical and chemical properties[7], but also has good dispersion, high mechanical strength and excellent lubrication performance. Therefore, in order to improve the tribological properties of UHMWPE, composite was prepared by ultrasonic dispersion and hot pressing. The results show the FG/UHMWPE composites have better wear resistance compared with FG/UHMWPE.

\section{Experiment}

Materials. M-II type UHMWPE powder (molecular weight, 200 million) was purchased from Shanghai Chemical Research Institute Tiandi Technology Development Co., Ltd.. Graphene oxide was purchased from Nanjing Xian Feng nano material technology Co., Ltd.. Zirconia ball (diameter, $5 \mathrm{~mm}$; rough degree, $0.04 \mu \mathrm{m}$ ) was purchased from Shanghai Xinmao precision ceramic technology Co., Ltd.. Hydrofluoric acid ( $\mathrm{w}=40 \%$ ), anhydrous ethanol, nitrate, sodium chloride were purchased 
from the Sinopharm Chemical Reagent Co., Ltd..

Preparation of FG/UHMWPE Composites. FG with high fluorine content was synthesized according to the literature $1.50 \mathrm{mg}$ graphene oxide sample was dispersed into $50 \mathrm{ml}$ ultrapure water by ultrasonic for $30 \mathrm{~min}$, then $5 \mathrm{ml}$ hydrofluoric acid $(\mathrm{w}=40 \%)$ and nitrate were added in the former dispersion. Then the mixture was shifted into a $100 \mathrm{ml}$ thermal water kettle with Teflon liner, and the The hydrothermal reaction ws carried out at 180 for $12 \mathrm{~h}$. After the reaction, the reaction liquid under $50{ }^{\circ} \mathrm{C}$ to obtain FG sample. A certain amount of FG powder was dispersed into $40 \mathrm{ml}$ ethanol with the aid of ultrasonic for 30 min. 2 g UHMWPE powder was added into the former suspension to get mixed liquid, and the mixed liquid was stirred for $60 \mathrm{~min}$. Then, ethanol in the mixed liquid evaporated to get mixed powder. The mixed powder was treated by thermal forming at $195^{\circ} \mathrm{C}$ and $10 \mathrm{Mpa}$ to obtian FG/UNMWPE composite sample. By using the method, FG/UNMWPE composite samples with the FG content of $0 \%, 0.1 \%, 0.3 \%, 0.5 \%$ and $1 \%$ were prepared respectively.

Characterization of FG/UHMWPE Composites. Microhardness testing was carried out on a microhardness tester (Shanghai Hengyi Precision Instrument Co., China), with a load of $10 \mathrm{~N}$ for 5 s. In order to ensure the accuracy of the experimental results, each FG/UHMWPE composite sample were test averaged ten points. After completion of the experiment using software Origin8.0, calculated on the basis of the test sample $\mu=\mathrm{F} / \mathrm{N}$ and plotted the coefficient of friction (coefficient of friction, $\mathrm{COF}$ ) and its stable phase $\mathrm{COF}$ average. At the same time according to the equation $\mathrm{K}=$ $\mathrm{V} / \mathrm{LS}$ alculate the wear rate (Wear rate, WR). Where $\mu$ is the coefficient of friction; $\mathrm{F}$ is the frictional force in $\mathrm{N} ; \mathrm{N}$ is the normal load force in $\mathrm{N}$; $\mathrm{K}$ is the wear rate in $\mathrm{mm} 3 /(\mathrm{Nm})$; $\mathrm{V}$ is the volume of the amount of wear in $\mathrm{mm} 3 /(\mathrm{N} \bullet \mathrm{m}): \mathrm{mm} 3$; $\mathrm{L}$ is sliding distance in $\mathrm{mm}$; $\mathrm{S}$ is the surface of the test the wear scar cross-sectional area in mm2.. Worn surface of FG/UHMWPE Composites is observed by SEM morphology.

\section{Results and discussion}

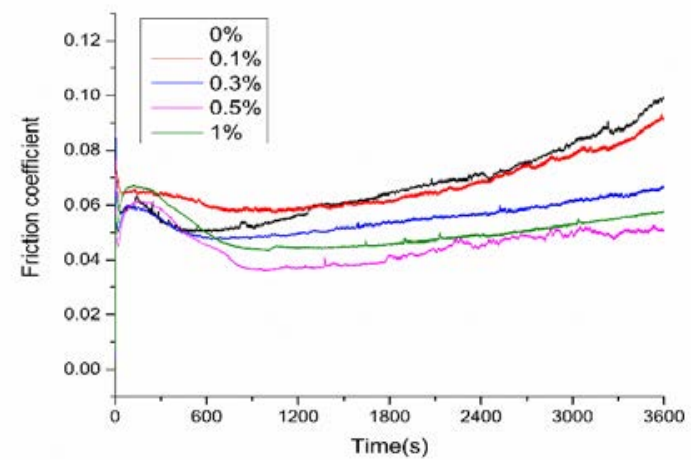

Fig.1 Variations of friction coefficient of the FG/UHMWPE composites with time

Figure 1 shows that the friction coefficient of the friction pair which is composed of the FG/UHMWPE composite material and zirconia is changed over time. It can be seen that the friction coefficient of FG/UHMWPE composites is lower, and the friction coefficient is decreased with the increase of FG content. This is due to the good self lubrication performance of FG. 


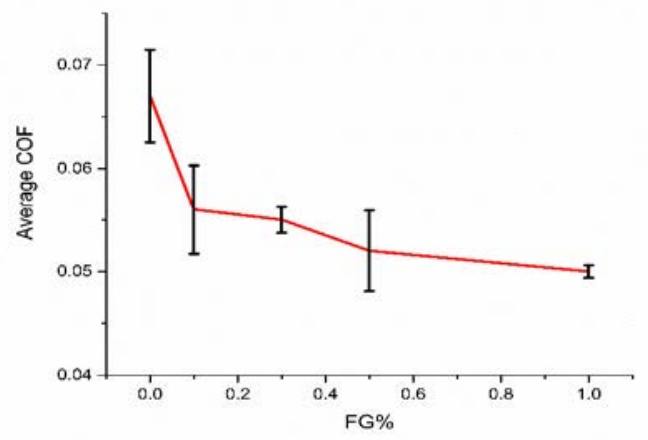

Fig.2 The average coefficient of friction of different FG/UHMWPE composites

Figure 2 shows the average friction coefficient of different composite materials, which can be seen that the addition of FG reduces the friction coefficient of UHMWPE. This is due to the good lubricating properties of fluorinated graphene, which is similar to the variation trend of the friction coefficient of the CNT/UHMWPE composite / zirconia friction pair[8-11].

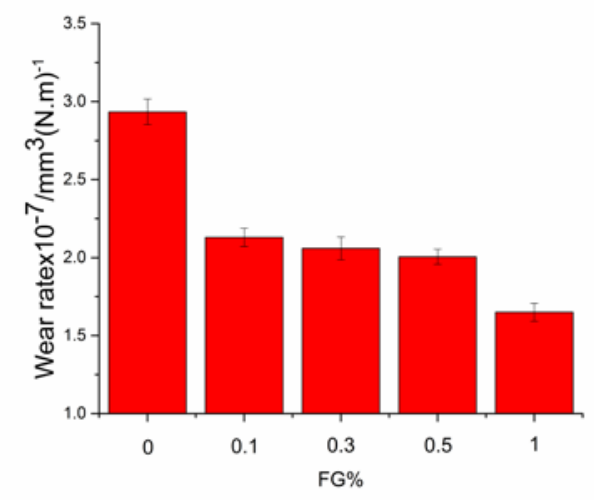

Fig.3 Variations volume wear rate of the FG/UHMWPE composites with FG content

Figure 3 is the change of volume wear rate of FG/UHMWPE composites with different FG content in the end of the wear test after the end of the experiment. It can be seen that the wear rate of pure UHMWPE in BAS lubricant is about $2.7 \times 10-7 \mathrm{~mm} 3 \cdot(\mathrm{N} \cdot \mathrm{m})-1$, with the increasing of FG content, the volume wear rate of FG/UHMWPE composites decreased obviously. When the content of FG was 1\%, the volume wear rate decreased by about $30 \%$, which indicated that the wear resistance of the composites was significantly improved by the addition of FG.

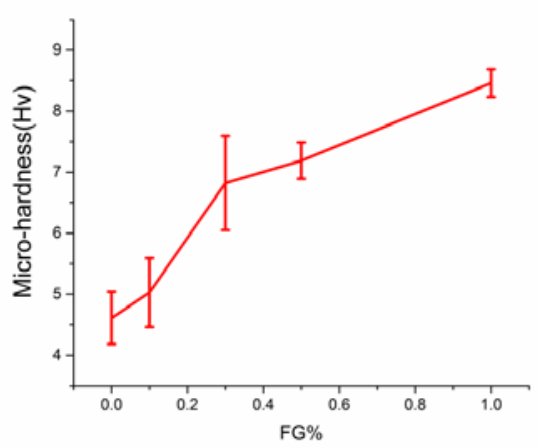

Fig.4. Variation of microhardness of the FG/UHMWPE composites with FG content

The micro hardness is the ability to resist the pressure from the other hard objects, and the wear resistance of the material is directly displayed on the other hand. Figure 4 is a FG composite with different FG/UHMWPE content of the micro hardness analysis, it can be seen that with the increase 
of FG content, the microhardness of FG/UHMWPE composite materials significantly improved. After the addition of $0.1 \%$ FG/UHMWPE, the microhardness of FG composite has been increased by about $20 \%$. When the content of FG was $1 \%$, the microhardness of FG/UHMWPE composite increased by about $55 \%$.

Fig. 5 SEM images of FG/UHMWPE composites with different FG contents were followed by the friction and wear tests. From the figure we can see pure UHMWPE surface wear is serious, there is kind of furrow breakage, adhesive wear. With the increase of the content of FG/UHMWPE, the wear of the surface of FG composites decreases gradually. Pure UHMWPE will produce debris due to its low hardness in the process of friction With the addition of UHMWPE, the surface hardness of FG gradually increased, and the ability to resist plastic deformation of composite materials was improved. On the one hand, the FG can provide the support of the fulcrum, the transfer of the scattered stress, and thus significantly enhance the wear resistance of the composite materials. On the other hand, the UHMWPE is a single chain macromolecular structure, and the combination of FG and the formation of a three-dimensional network structure, which greatly enhances the composite of the micro hardness. The combination of 1-5 can be seen that the addition of FG can improve the tribological properties of UHMWPE.

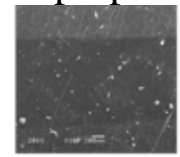

a $w(\mathrm{FG})=0 \%$

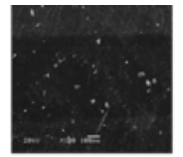

$\mathrm{b} w(\mathrm{FG})=0.1 \%$

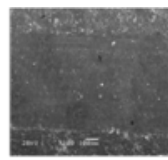

$\mathrm{c} w(\mathrm{FG})=0.3 \%$

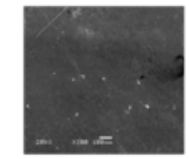

$\mathrm{d} w(\mathrm{FG})=0.5 \%$

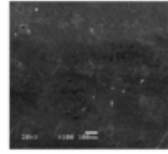

e $w(\mathrm{FG})=1 \%$

Fig.5. SEM micrographs of scratches of different FG/UHMWPE composites after the wear test

\section{Conclusions}

i) The addition of FG can significantly improve the microhardness of UHMWPE.

ii) The friction coefficient of FG/UHMWPE composites decreases with the pure UHMWPE, and the volume wear rate decreases obviously.

\section{Acknowledgement}

In this paper, the research was sponsored by the National Natural Science Foundation of China (Project No.51475447)

\section{References}

[1] Turos A, Abdul-Kader A M, Grambole D, et al. The effects of ion bombardment of ultra-high molecular weight polyethylene. Nuclear Instruments and Methods in Physics Research Section B: Beam Interactions with Materials and Atoms, 2006, 249(1): 660-664.

[2] Krzypow D J, Rimnac C M. Cyclic steady state stress-strain behavior of UHMW polyethylene. Biomaterials, 2000, 21(20): 2081-2087.

[3] Satyanarayana N, Sinha S K, Ong B H. Tribology of a novel UHMWPE/PFPE dual-film coated onto Si surface. Sensors and Actuators A: Physical, 2006, 128(1): 98-108. 
[4] Ohta M, Hyon S H, Tsutumi S. Control of crystalline orientation to enhance the wear resistance of ultra-high molecular weight polyethylene crystallization cups for artificial joints. Wear, 2003, 255(7): 1045-1050.

[5] McKellop H A. Bearing surfaces in total hip replacements: state of the art and future developments. Instructional course lectures, 2000, 50: 165-179.

[6] Xie X L, Tang C Y, Chan K Y Y, et al. Wear performance of ultrahigh molecular weight polyethylene/quartz composites. Biomaterials, 2003, 24(11): 1889-1896.

[7] Ma Xiaochen. The preparation of fluorinated Graphene and chemical reduction method for preparation of graphene. Nanjing: Nanjing University of Science and Technology, 2012.

[8] Geringer J, Tatkiewicz W, Rouchouse G. Wear behavior of PAEK, poly (aryl-ether-ketone), under physiological conditions, outlooks for performing these materials in the field of hip prosthesis. Wear, 2011, 271(11): 2793-2803.

[9] AN Y, TAI Z, YAN X, et al. Tribological Property of GO/UHMWPE Composites under the Lubrication of Bovine Serum Albumin. Tribology, 2012, 32(6): 533-537.

[10] AN Y, PEI J, YAN X, et al. The Tribological Property of UHMWPE under the lubrication of Sodium Hyaluronate Reinforced by Graphene Oxide. Tribology, 2014, 34(2): 113-119.

[11] Yang D, Qu S, Huang J, et al. Characterization of alendronate sodium-loaded UHMWPE for anti-osteolysis in orthopedic applications. Materials Science and Engineering: C, 2012, 32(2): 83-91. 